\title{
C-reactive protein, ESR, and klebsiella in ankylosing spondylitis
}

\author{
P. COWLING, R. EBRINGER, ${ }^{1}$ D. CAWDELL, ${ }^{1}$ M. ISHII,
} AND A. EBRINGER

From the Immunology Unit, Department of Biochemistry, Queen Elizabeth College, London, and the ${ }^{1}$ Departments of Microbiology and Rheumatology, Middlesex Hospital, London

SUMMARY Serum C-reactive protein (CRP) and erythrocyte sedimentation rate (ESR) were measured on 469 occasions in 149 patients with ankylosing spondylitis who had also been investigated for faecal carriage of klebsiella/enterobacter species on the same occasions. Raised values of CRP and ESR correlated with each other $(\mathrm{P}<0 \cdot 001)$ as well as with clinically assessed active disease episodes $(\mathrm{P}<0 \cdot 001)$. Patients with positive cultures for klebsiella were found to have higher mean values for CRP and ESR than those with negative cultures $(P<0.025)$. CRP appears to be a useful marker of disease activity in ankylosing spondylitis.

An association between clinically active inflammatory disease in ankylosing spondylitis (AS) and the presence in faeces of a Gram-negative microorganism, Klebsiella pneumoniae, has been reported (Ebringer et al., 1977; Ebringer et al., 1978). Disease activity in those studies was assessed for each individual visit on the basis of the patient's assessment and the doctor's findings, laboratory and faecal culture results only being available later. Both ESR and CRP have been shown to be useful indicators of disease activity in other rheumatic diseases such as rheumatoid arthritis (Amos et al., 1977).

The function of CRP is unknown. It is produced in the liver and found only in low levels in normal human serum. High levels are found during acute infection, tissue injury, or inflammatory disease. The molecule is capable of activating the complement cascade and initiate both the opsonic and lytic potentials of this system (Siegel et al., 1975; Osmand et al., 1975; Mortensen et al., 1976). These characteristics suggest that CRP may play an important role in the mediation of inflammatory responses.

The value of CRP in ankylosing spondylitis has not been previously examined except in 1 early study (Glyn and Irving, 1959). In this investigation we set out to determine if ESR and CRP correlated or

Accepted for publication 29 January 1979

Correspondence to Dr R. Ebringer, Department of Rheumatology, Middlesex Hospital, Arthur Stanley House, Tottenham Street, London W1P 9PG. contributed to our understanding of disease in ankylosing spondylitis when compared both to the clinically assessed disease activity and the results of faecal culture.

\section{Patients and methods}

\section{PATIENT SELECTION}

Serum samples were obtained from 149 patients attending the Middlesex Hospital AS clinics on 469 occasions. Each patient had an average of 3 visits (range 1-10). The patient's samples were selected randomly from a bank of serum samples obtained at regular visits over the past 2 years. The diagnosis of AS was made by the New York criteria (Bennet and Wood, 1968). Five patients with psoriatic spondylitis and 6 who had inflammatory bowel disease with spondylitis were included. Of 125 patients tissue typed 117 carried the B27 antigen. Two patients with colitic spondylitis and 2 with psoriatic spondylitis did not carry the B27 antigen. Six patients were included who had normal or suspicious sacroiliac $x$-ray changes with low back pain. All were HLA B27 positive and most had a positive family history, a positive sacroiliac scintiscan, or peripheral joint involvement. The mean age of all patients was 34.5 years (range 13-72), with a mode of 26 years. There were 33 female patients.

ASSESSMENT OF DISEASE ACTIVITY

Assessment was done at the time of the patient's 
visit and before the results of CRP, ESR, and faecal culture were available.

Assessment of active disease was made on the basis of acute peripheral manifestations such as active synovitis, dactylitis, joint or spinal tenderness, or acute anterior uveitis. If a patient had an effusion which was not tender or inflamed and which had been present for months, this was more likely to be assessed as probably active disease. Assessment of active disease was also made on the basis of recent (within the past 4 weeks) exacerbation of peripheral or spinal symptoms. Anti-inflammatory drug intake had usually increased, or the maintained dose had failed to control symptoms. There was nearly always worsening of the patient's self-rated visual analogue scale of pain.

Patients with probably active disease usually had symptoms which were stable or constant and were well controlled with anti-inflammatory therapy. Alternatively, they may have had more severe symptoms and signs in the previous few months and now appeared to be improving. Short fluctuations in symptoms in general were disregarded.

Patients assessed as having inactive disease were either symptomless or had only occasional mild or short-lived symptoms. They usually did not require medication or, if they did, the dose was regular and effective.

\section{CONTROL SUBJECTS}

Sixty healthy control subjects having no previous history of arthritis or back pain and not in a hospital environment were bled for estimation of serum CRP.

CRP ESTIMATIONS

The Mancini radial immunodiffusion technique with commercial antisera (Hoechst) was used. Each estimation was the mean of at least 2 separate measurements. All measurements were carried out without knowledge of the patient's clinical status.

\section{ESR ESTIMATIONS}

Blood for ESR was taken on the day of assessment and measured by the Westergren method. The normal hospital service was used and there was no special identification for the ESR samples measured.

\section{FAECAL CULTURES}

Faecal specimens were collected and cultured as described previously (Ebringer et al., 1978). Faecal cultures were read without knowledge of the patient's clinical status.

\section{PATIENTS ON ANTIBIOTICS}

Serum samples used also included patients who are on a trial of an antibiotic, Co-trimoxazole, at the time of assessment. These results were analysed separately when disease activity was assessed.

\section{Results}

\section{CRP AND DISEASE ACTIVITY}

The mean serum CRP level in active patients was $32.78 \pm 5.33 \mu \mathrm{g} / \mathrm{ml}$ (mean \pm standard error of the mean), in probably active patients it was $27.64 \pm 1.99 \mu \mathrm{g} / \mathrm{ml}$, and in inactive patients it was $13.22 \pm 0.82 \mu \mathrm{g} / \mathrm{ml}$ (Fig. 1). The difference in mean levels between active and inactive patients $(t=6 \cdot 62$, $\mathrm{P}<0.001$ ), as well as between probably active and inactive patients $(t=7 \cdot 33, \mathrm{P}<0 \cdot 001)$ was statistically significant.

The mean serum CRP level in 60 healthy control subjects was $3.57 \pm 0.61 \mu \mathrm{g} / \mathrm{ml}$. Of the 60 control subjects $31(52 \%)$ had zero values for their serum CRP levels.

The difference in mean serum CRP levels between the 3 different disease activity groups in ankylosing spondylitis compared to healthy control subjects was statistically significant: active $(t=7 \cdot 17, \mathrm{P}<0.001)$ probably active $(t=6 \cdot 82, \mathrm{P}<0.001)$, and inactive $(t=5 \cdot 68, \mathrm{P}<0 \cdot 001)$.

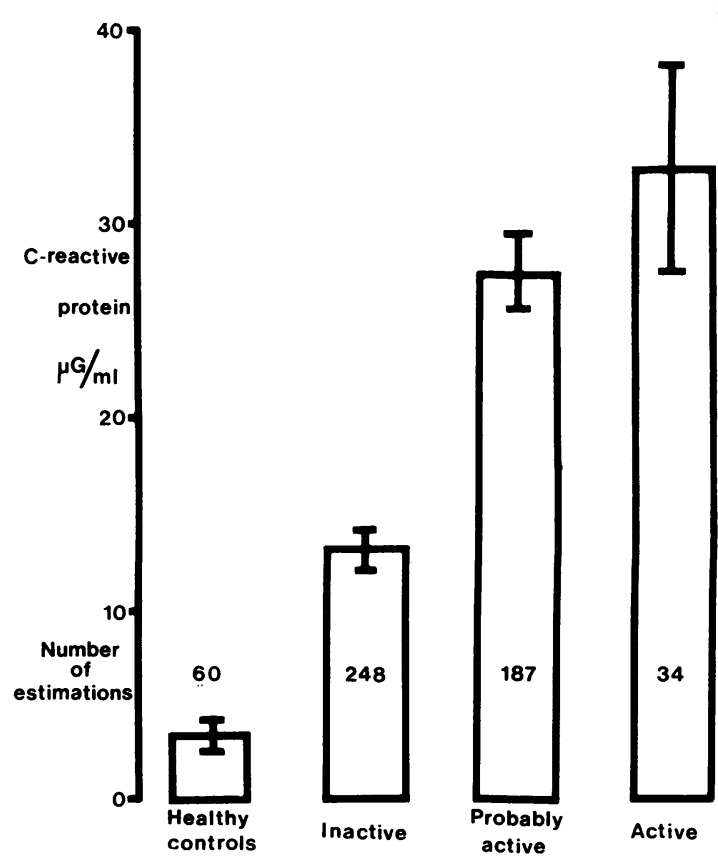

Fig. 1 Mean serum CRP levels in healthy control subjects and patients with ankylosing spondylitis during different phases of disease activity. Bars indicate standard errors of the mean. 
ESR AND DISEASE ACTIVITY

The mean ESR in active patients was $37 \cdot 10 \pm 5.99$ $\mathrm{mm} / \mathrm{h}$ Westergren (mean \pm standard error of the mean), in probably active patients $25.99 \pm 1.65$ $\mathrm{mm} / \mathrm{h}$, and in inactive patients $14.28 \pm 0.90 \mathrm{~mm} / \mathrm{h}$ (Fig. 2). The differences in mean ESR value between active and inactive patients $(t=6.96, \mathrm{P}<0.001)$ and probably active and inactive patients $(t=6 \cdot 62$, $\mathbf{P}<0.001)$ were statistically significant.

Not only was there a correlation between mean CRP and mean ESR levels in various phases of disease activity, but individual values of high or low ESR were closely correlated with high or low values in serum CRP irrespective of disease activity $(r=+0.6947, D F=430, P<0.001)$.

\section{ISOLATION OF KLEBSIELLA AND DISEASE} ACTIVITY

The results of culture investigations in patients with active, probably active, and inactive ankylosing spondylitis are summarised in Table 1. Klebsiella

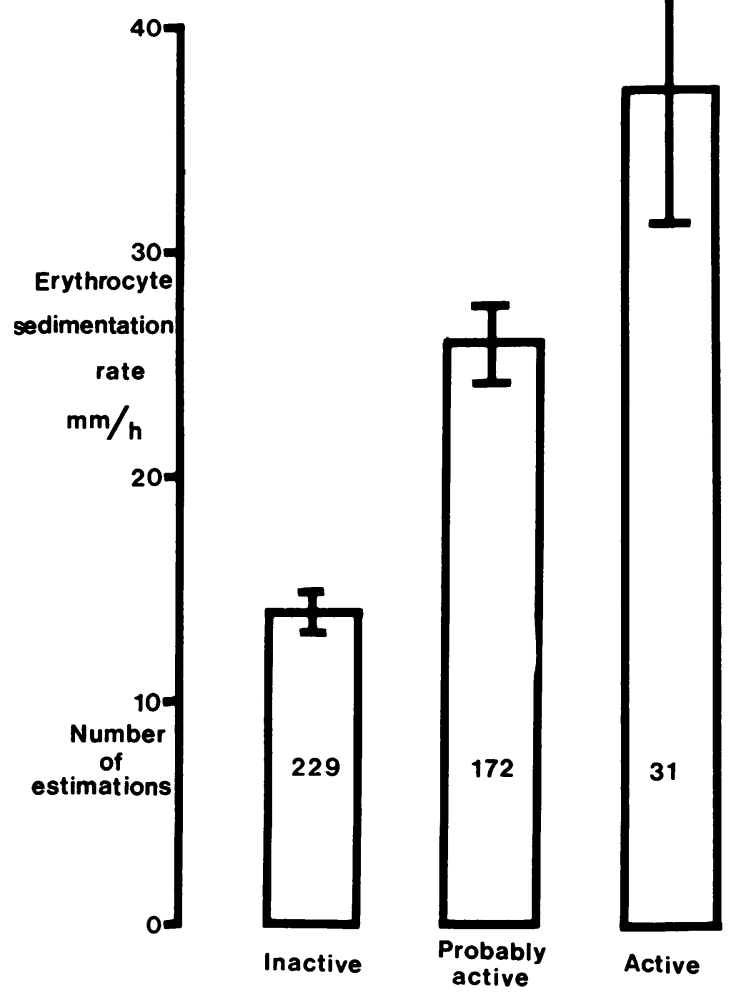

Fig. 2 Mean ESR levels in patients with ankylosing spondylitis, during different phases of disease activity. Bars indicate standard errors of the mean
Table 1 Frequency of isolation of klebsiella enterobacter species in patients with active, probably active, and inactive ankylosing spondylitis. Numbers in brackets indicate number of episodes where patients were on antibiotics

\begin{tabular}{|c|c|c|c|}
\hline & Active & $\begin{array}{l}\text { Probably } \\
\text { active }\end{array}$ & Inactive \\
\hline $\begin{array}{l}\text { Culture positive } \\
\text { Culture negative } \\
\text { Percentage of positive }\end{array}$ & $\begin{array}{l}11(0) \\
14(6)\end{array}$ & $\begin{array}{c}45(6) \\
104(34)\end{array}$ & $\begin{array}{c}42(4) \\
154(24)\end{array}$ \\
\hline $\begin{array}{l}\text { cultures in all patients } \\
\text { Percentage of positive } \\
\text { cultures in patients not } \\
\text { taking antibiotics }\end{array}$ & $44 \%$ & $30 \%$ & $21 \%$ \\
\hline
\end{tabular}

species of micro-organisms were isolated more frequently from patients during active or probably active phases than during inactive phases of ankylosing spondylitis. The difference between the isolation rate of klebsiella in active patients compared to inactive patients was statistically significant (Yates's correction, chi-square $=5 \cdot 02, \mathrm{P}<0.025$ ), but not significant when probably active patients were compared to inactive patients (Yates's correction, chi-square $=3 \cdot 01$, NS).

This study, however, included some patients who were taking antibiotics. If the episodes where patients were taking antibiotics are excluded (Table 1), the frequency of isolation of klebsiella micro-organisms increases in all 3 categories of disease activity. The difference between the isolation of klebsiella in both active (Yates's correction, chi-square $=\mathbf{9 \cdot 1 8}$, $\mathrm{P}<0.005$ ) and probably active (Yates's correction, chi-square $=5.07, P<0.025$ ) patients not taking antibiotics is statistically significant when compared to a similar group of inactive patients.

\section{CRP AND ESR IN PATIENTS WITH AND} WITHOUT KLEBSIELLA

All the episodes in patients in whom klebsiella was isolated from faecal cultures were grouped together irrespective of disease activity and compared to all the episodes when no klebsiella was isolated. The mean serum CRP in patients having klebsiella was $25 \cdot 17 \pm 2.91 \mu \mathrm{g} / \mathrm{ml}$ (mean \pm standard error of the mean), while in patients not having klebsiella it was $18.57 \pm 1.21 \mu \mathrm{g} / \mathrm{ml}$ (Fig. 3), and this difference is statistically significant $(t=2 \cdot 48, \mathrm{P}<0 \cdot 01)$. The mean ESR in patients having klebsiella in their faeces was $24 \cdot 15 \pm 2.56 \mathrm{~mm} / \mathrm{h}$, while in patients not having klebsiella it was $18.78 \pm 1.13 \mathrm{~mm} / \mathrm{h}$ (Fig. 4), and this difference is statistically significant $(t=2 \cdot 21, \mathrm{P}<0 \cdot 025)$.

\section{Discussion}

Our findings show that patients with AS have raised 


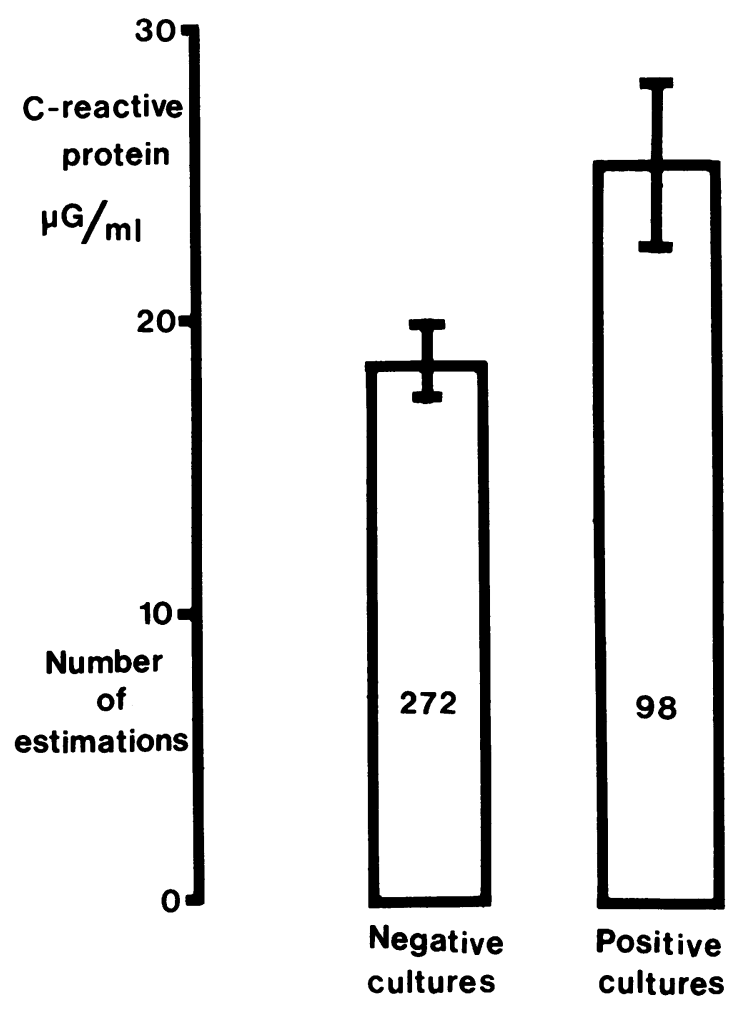

Fig. 3 Mean CRP levels in patients with positive or negative faecal cultures for klebsiella/enterobacter species. Bars indicate standard errors of the mean

CRP and ESR values comparable to the values found in patients with rheumatoid arthritis (Amos et al., 1978). Those patients with clinically assessed active disease have higher mean CRP and ESR results than patients with clinically less active disease. Moreover the presence of klebsiella/enterobacter in the faeces is associated with a higher mean CRP and ESR irrespective of the clinically assessed disease activity. Thus there is an association between the presence of klebsiella in AS patients and active disease as assessed independently by clinical and laboratory criteria.

Our clinical assessment of disease activity was deliberately aimed at selecting episodes of acute inflammatory disease whether these were episodes of acute anterior uveitis, peripheral arthritis, spinal disease, or a combination of the 3 . It is more difficult to assess such active episodes when the symptoms relate to pelvic or spinal disease alone, and it is for this reason that recent worsening of symptoms played a major role in guiding the grading of disease activity.

Clinical observation suggests that AS is not an
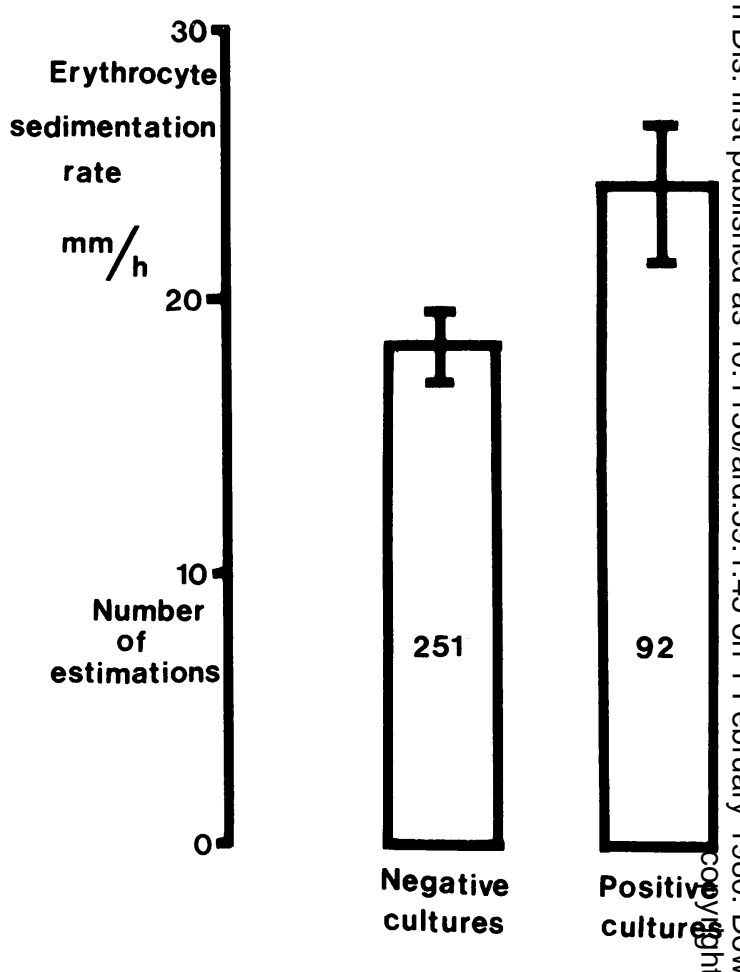

Fig. 4 Mean ESR levels in patients with positive or negative faecal cultures for klebsiella/enterobacter species. Bars indicate standard errors of the mean

inexorable progressive disease and that it follows a pattern of exacerbations and remissions. The sacroiliitis and spinal disease as evidenced by limitation of movement and radiographic changes would, under this hypothesis, more probably reflect the gradual accumulation of partially irreversible, chronic secondary changes due to the initial inflammatory episodes. It is possible that each major flare-up of disease in the patients, whether acute anterior uveitis, peripheral arthritis, or spinal inflammation, may be due to reinfection or $\frac{D}{O}$ rechallenge by the agent or agents responsible for triggering the inflammation. This hypothesis there- of fore suggests that ankylosing spondylitis and the episodes of acute anterior uveitis associated with AS and HLA B27 are due to a form of reactive arthritis $\omega$ or reactive inflammation, probably immunologically mediated. Klebsiella pneumoniae may be one $\bullet$ of the micro-organisms which trigger off such $\mathbb{\Phi}$ exacerbations of inflammatory joint or eye disease.

The best model of reactive arthritis is acute rheumatic fever. In this disease there is a clear difference between the acute rheumatic symptoms $\stackrel{\odot}{\oplus}$

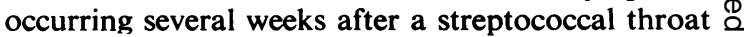


infection and the disability due to incompetent or stenosed cardiac valves of chronic rheumatic heart disease. We would suggest that there is an analogy here between the acute inflammatory episode and the chronic diseased valves and the acute inflammatory episodes of AS and the sacroiliitis and spondylitis of established disease. The search for an aetiological agent in rheumatic fever would have been unrewarding if only patients with established rheumatic heart disease were studied. Similarly we anticipated that the 'active disease' or acute inflammatory episodes in AS were the times to search for potential aetiological agents.

The pathogenesis of AS is still unclear. If we assume that an infective micro-organism is involved, the pathological lesion of AS could be produced either directly by infection or by release of toxins or bacterial debris from the implicated gut microorganisms such as klebsiella/enterobacter species, salmonella, shigella, or Yersinia enterocolitica. Another possible mechanism is that partially cross-reacting antibodies binding to self antigens are produced, triggering the complement cascade and producing localised inflammation at a site distal to the site of infection. All 3 mechanisms may play a role. We favour the cross-reactivity mechanism (Ebringer, 1978; Young et al., 1978).

In rheumatic fever, the infection is located in the upper respiratory tract, and inflammatory damage mediated by cross-reacting antistreptococcal antibodies occurs in brain and endomyocardium, producing chorea and rheumatic heart disease. In ankylosing spondylitis the infection might be located in the lower gastrointestinal tract, and inflammatory damage, triggered by cross-reacting antimicrobial antibodies, might occur in joint, spine, and eyeball, producing sacroiliitis and uveitis. Whether such antibody can be demonstrated remains to be seen.

We thank Dr G. Panayi for his helpful discussions during this study and Mrs Coralie Padamsey for typing the manuscript.

\section{References}

Amos, R. S., Constable, T. J., Crockson, R. A., Crockson, A. P., and McConkey, B. (1977). Rheumatoid arthritis: relation of serum C-reactive protein and erythrocyte sedimentation rates to radiographic changes. British Medical Journal, 1, 195-197.

Amos, R. S., Crockson, R. A., Crockson, A. P., Walsh, L., and McConkey, B. (1978). Rheumatoid arthritis: Creactive protein and erythrocyte sedimentation rate during initial treatment. British Medical Journal, 1, 1396.

Bennet, P. H., and Wood, P. H. N. (1968). In Population Studies of the Rheumatic Diseases, p. 456. Edited by by P. H. Bennet and P. H. N. Wood. Excerpta Medica Foundation: Amsterdam.

Ebringer, A. (1978). The link between genes and disease. New Scientist, 79, 865-867.

Ebringer, R., Cooke, D., Cawdell, D. R., Cowling, P., and Ebringer, A. (1977). Ankylosing spondylitis: Klebsiella and HL-A B27. Rheumatology and Rehabilitation, 16, 190-195.

Ebringer, R. W., Cawdell, D. R., Cowling, P., and Ebringer, A. (1978). Sequential studies in ankylosing spondylitis: association of Klebsiella pneumoniae with active disease. Annals of the Rheumatic Diseases, 37, 146-151.

Glyn, J. H., and Irving, M. (1959). Laboratory investigations in the diagnosis of rheumatic diseases. Annals of Physical Medicine, 5, 1-9.

Mortensen, R. F., Osmand, A. P., Lint, T. F., and Gewurz, H. (1976). Interaction of C-reactive protein with lymphocytes and monocytes, complement dependent adherence and phagocytosis. Journal of Immunology, 117, 774-781.

Osmand, A. P., Mortensen, R. F., Siegel, J., and Gewurz, H. (1975). Interactions of C-reactive protein with the complement system. III. Complement-dependent passive hemolysis initiated by CRP. Journal of Experimental Medicine, 142, 1065-1077.

Siegel, J., Osmand, A. P., Wilson, M. F., and Gewurz, H. (1975). Interactions of C-reactive protein with the complement system. II. C-reactive protein mediated consumption of complement by poly-L-lysine polymers and other polycations. Journal of Experimental Medicine, 142, 709-721.

Young, C. R., Ebringer, A., and Archer, J. (1978). Immune response inversion following hyperimmunization: A possible mechanism in the pathogenesis of HLA-linked diseases. Annals of the Rheumatic Diseases, 37, 152-158. 